\title{
Optimizing Cooperative Cognitive Radio Networks with Opportunistic Access
}

\author{
Ammar Zafar, ${ }^{1}$ Mohamed-Slim Alouini, ${ }^{1}$ Yunfei Chen, ${ }^{2}$ and Redha M. Radaydeh ${ }^{3,4}$ \\ ${ }^{1}$ Electrical Engineering Program, KAUST, Al Khawarizmi Applied Mathematics Building 1, Mail Box 2675, Makkah Province, \\ Thuwal 23955-6900, Saudi Arabia \\ ${ }^{2}$ School of Engineering, University of Warwick, Coventry, CV4 7AL, UK \\ ${ }^{3}$ Electrical Engineering Department, KAUST, Thuwal 23955-6900, Saudi Arabia \\ ${ }^{4}$ Department of Electrical and Computer Engineering, Texas A\&M University, Texas A\&M Engineering Building, \\ Education City, Doha, Qatar
}

Correspondence should be addressed to Ammar Zafar, ammar.zafar@kaust.edu.sa

Received 9 January 2012; Revised 19 March 2012; Accepted 20 March 2012

Academic Editor: Enrico Del Re

Copyright (C) 2012 Ammar Zafar et al. This is an open access article distributed under the Creative Commons Attribution License, which permits unrestricted use, distribution, and reproduction in any medium, provided the original work is properly cited.

\begin{abstract}
Optimal resource allocation for cooperative cognitive radio networks with opportunistic access to the licensed spectrum is studied. Resource allocation is based on minimizing the symbol error rate at the receiver. Both the cases of all-participate relaying and selective relaying are considered. The objective function is derived and the constraints are detailed for both scenarios. It is then shown that the objective functions and the constraints are nonlinear and nonconvex functions of the parameters of interest, that is, source and relay powers, symbol time, and sensing time. Therefore, it is difficult to obtain closed-form solutions for the optimal resource allocation. The optimization problem is then solved using numerical techniques. Numerical results show that the allparticipate system provides better performance than its selection counterpart, at the cost of greater resources.
\end{abstract}

\section{Introduction}

The ever increasing wireless communication networks have put great stress on the already limited spectrum. Due to the fixed spectrum allocation policy, only the licensed users, otherwise known as primary users, are able to access the licensed spectrum. Additionally, the Federal Communications Commission (FCC) task force highlighted in their report the fact that at any given time only $2 \%$ of the spectrum is being used [1]. Therefore, ensuring better spectrum usage is of paramount importance.

Cognitive radios have been proposed to resolve this issue [2]. In cognitive radio, the unlicensed users, otherwise known as secondary users, first sense the licensed bands for spectrum holes (parts of the licensed spectrum which are not being employed by the primary users at some time in certain geographical location) [3]. Then, if a spectrum hole is found, the secondary users transmit data to the intended destination. However, the secondary user has to be careful so as not to cause interference to the primary user. The two stages of spectrum sensing and data transmission are related and for optimal performance must be optimized jointly. This is due to the probability of detection, $P_{d}$, and probability of false alarm, $P_{f}$, associated with spectrum sensing. If the secondary user with probability $P_{f}$ misses a spectrum hole, then it will keep silent and miss an opportunity to transmit, reducing throughput. However, if a transmission from the primary is missed, with probability $1-P_{d}$, then the secondary user transmits and causes interference to the primary user. Moreover, due to interference, the signalto-noise-ratio (SNR) of the secondary user also decreases, decreasing the throughput and the symbol error rate (SER). Resource allocation that optimizes this sensing-throughput tradeoff has been discussed in [4]. Other optimal resource allocation algorithms for cognitive radio networks have been discussed in [5]. More specifically, in [5], the authors considered a multiband system and considered the two cases of sensing-based spectrum sharing and opportunistic 
spectrum access. However, both the above-mentioned works maximize the throughput.

In this paper, optimal resource allocation is discussed to minimize the SER. In order to achieve minimum SER, cooperation is introduced into the system as it decreases the SER due to diversity [6]. Hence, the transmitting secondary user now, upon sensing a spectrum hole, transmits to the relays as well as the destination. Power allocation for relayassisted cognitive radio networks has been discussed in [7-15]. These works proposed strategies to maximize the throughput for a cognitive relay network that is allowed to share the frequency band with the primary user. Thus, they did not consider spectrum sensing for opportunistic access. Here, we consider an opportunistic system with amplifyand-forward (AF) relays. Full channel state information is assumed at the central controller which performs the resource allocation. Firstly, an all-participate (AP) system is discussed and it is shown that the optimization problem is nonconvex and hence cannot be solved using analytical means. It is then noted that the AP system is limited due to the systems resources being orthogonally distributed. To rectify this, a selection scheme is proposed and the optimal resource allocation, in this case, is discussed.

The rest of the paper is organized as follows. Section 2 gives the system model. The AP system is considered in Section 3. Section 4 details selective relaying. Numerical results are discussed in Section 5. Finally, Section 6 concludes the paper.

\section{System Model}

Consider a cognitive radio network in which the secondary source utilizes $m$ relays to send data to the secondary destination as shown in Figure 1. The secondary network only has opportunistic access to the licensed spectrum. Therefore, it needs to perform spectrum sensing. The source performs the spectrum sensing and then transmits information to the relays and destination if it finds a "spectral hole" in the first time slot. The relays then forward the received signal to the destination after amplification. In this paper narrowband channel is assumed. The source and the relays can use frequency orthogonal channels to avoid causing interference to each other in wideband channels. For ease of analysis, we consider time orthogonal channels here. Hence, a total of $m+1$ time slots are used.

2.1. Received Signal Model. Based on the spectrum sensing result, there are two possible received signal models.

2.1.1. Without Interference from the Primary User. In this scenario, with probability $1-P_{f}$, where $P_{f}$ is the probability of false alarm, the source correctly detects the presence of a "spectral hole" and transmits. The signals received at the destination and the relays are

$$
\begin{aligned}
& y_{r_{t}}=\sqrt{E_{\mathrm{ST}}} h_{r_{t}} s+n_{r_{t}}, \\
& y_{i_{t}}=\sqrt{E_{\mathrm{ST}}} h_{i_{t}} s+n_{i_{t}},
\end{aligned}
$$

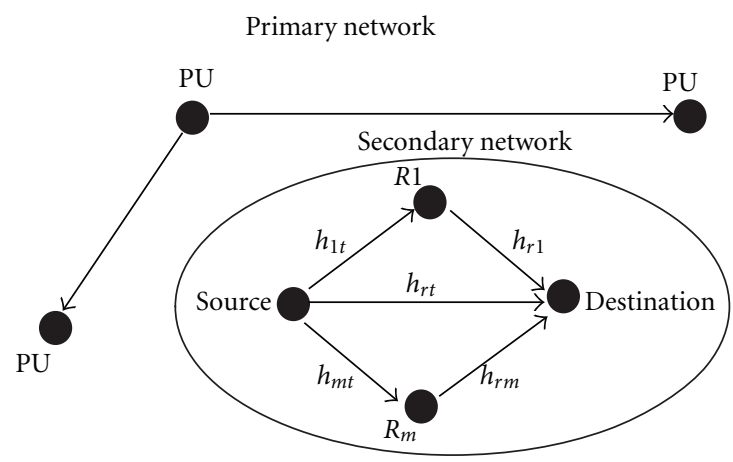

FIGURE 1: Primary and secondary networks.

where $s$ is the zero-mean and unit-energy transmitted symbol, $E_{\mathrm{ST}}$ is the source energy, $h_{r_{t}}$ is the channel response between the source and the destination, $h_{i_{t}}$ is the channel response between the $i$ th relay and the source, and $n_{r_{t}} \sim$ $\mathrm{CN}\left(0, \sigma_{n_{r_{t}}}^{2}\right)$ and $n_{i_{t}} \sim \mathrm{CN}\left(0, \sigma_{n_{i_{t}}}^{2}\right)$ are the complex Gaussian noise samples. The relay, after normalization and amplification, forwards the received signal to the destination. The signal after normalization becomes

$$
s_{i}=\sqrt{\frac{E_{\mathrm{ST}}}{E_{\mathrm{ST}}\left|h_{i_{t}}\right|^{2}+\sigma_{n_{i_{t}}}^{2}}} h_{i_{t}} s+\sqrt{\frac{1}{E_{\mathrm{ST}}\left|h_{i_{t}}\right|^{2}+\sigma_{n_{i_{t}}}^{2}}} n_{i_{t}} .
$$

Therefore, the received signal at the destination from the $i$ th relay is

$$
y_{r_{i}}=\sqrt{E_{i}} h_{r_{i}} s_{i}+n_{r_{i}}
$$

where $h_{r_{i}}$ is the known channel response between the receiver and the $i$ th relay, $E_{i}$ is the $i$ th relay's energy, and $n_{r_{i}} \sim$ $\mathrm{CN}\left(0, \sigma_{n_{r_{i}}}^{2}\right)$ is the complex Gaussian noise. Substituting $s_{i}$ in (3) gives

$$
y_{r_{i}}=\sqrt{\frac{E_{\mathrm{ST}} E_{i}}{E_{\mathrm{ST}}\left|h_{i_{t}}\right|^{2}+\sigma_{n_{i_{t}}}^{2}}} h_{r_{i}} h_{i_{t}} s+\tilde{n}_{r_{i}}
$$

where $\tilde{n}_{r_{i}} \sim \mathrm{CN}\left(0, \tilde{\sigma}_{n_{r_{i}}}^{2}\right)$ and

$$
\tilde{\sigma}_{n_{r_{i}}}^{2}=\frac{E_{i}\left|h_{r_{i}}\right|^{2} \sigma_{n_{i_{t}}}^{2}}{E_{\mathrm{ST}}\left|h_{i_{t}}\right|^{2}+\sigma_{n_{i_{t}}}^{2}}+\sigma_{n_{r_{i}}}^{2}
$$

Writing the $m+1$ received signals in matrix form, one has

$$
\mathbf{y}=\mathbf{h} s+\mathbf{n}
$$


where

$$
\begin{aligned}
& \mathbf{y}= {\left[\frac{1}{\sigma_{n_{r_{t}}}} y_{r_{t}} \frac{1}{\widetilde{\sigma}_{n_{r_{1}}}} y_{r_{1}} \cdots \frac{1}{\tilde{\sigma}_{n_{r_{m}}}} y_{r_{m}}\right]^{T}, } \\
& \mathbf{h}=\left[\sqrt{\frac{E_{\mathrm{ST}}}{\sigma_{n_{r_{t}}}^{2}}} h_{r_{t}} \sqrt{\frac{E_{\mathrm{ST}} E_{1}}{\tilde{\sigma}_{n_{r_{1}}}^{2}\left(E_{\mathrm{ST}}\left|h_{1_{t}}\right|^{2}+\sigma_{n_{1_{t}}}^{2}\right)}}\right. \\
& \\
& \times h_{r_{1}} h_{1_{t}} \cdots \sqrt{\frac{E_{S T} E_{m}}{\widetilde{\sigma}_{n_{r_{m}}}^{2}\left(E_{\mathrm{ST}}\left|h_{m_{t}}\right|^{2}+\sigma_{n_{m_{t}}}^{2}\right)}} \\
&\left.\times h_{r_{m}} h_{m_{t}}\right]^{T}
\end{aligned}
$$

and $\mathbf{n}$ is a $(m+1)$ dimensional vector whose components are zero mean and unit variance complex Gaussian random variables. Therefore $\mathbf{n}$ is also complex Gaussian with mean vector of all zeros and covariance matrix being the identity matrix, $\mathbf{I}_{(m+1) \times(m+1)}$, that is $\mathbf{n} \sim \mathrm{CN}(\mathbf{0}, \mathbf{I})$.

2.1.2. With Interference from the Primary User. In this case, with probability $1-P_{d}$ where $P_{d}$ is the probability of detection, the source misses the transmission from the primary user and transmits, which causes interference. The signals at the destination from both the source and the relays now also include an interfering signal due to primary user activity is

$$
\begin{aligned}
& y_{r_{t}}=\sqrt{E_{\mathrm{ST}}} h_{r_{t}} s+n_{r_{t}}+y_{I_{r_{t}}}, \\
& y_{i_{t}}=\sqrt{E_{\mathrm{ST}}} h_{i_{t}} s+n_{i_{t}}+y_{I_{i_{t}}},
\end{aligned}
$$

where $y_{I_{r_{t}}}$ and $y_{I_{i_{t}}}$ are the interference signals.

Taking into account the fact that the source and relays have no knowledge of the interfering signal and adopting the same approach as previously, one can write

$$
y_{r_{i}}=\sqrt{\frac{E_{\mathrm{ST}} E_{i}}{E_{\mathrm{ST}}\left|h_{i_{t}}\right|^{2}+\sigma_{n_{i_{t}}}^{2}}} h_{r_{i}} h_{i_{t}} s+\hat{n}_{r_{i}}+\hat{y}_{I_{r_{i}}},
$$

where $\hat{n}_{r_{i}} \sim \mathrm{CN}\left(0, \hat{\sigma}_{n_{i}}^{2}\right)$ and

$$
\begin{gathered}
\hat{\sigma}_{n_{r_{i}}}^{2}=\frac{E_{i}\left|h_{r_{i}}\right|^{2} \sigma_{n_{i_{t}}}^{2}}{E_{\mathrm{ST}}\left|h_{i_{t}}\right|^{2}+\sigma_{n_{i_{t}}}^{2}}+\sigma_{n_{r_{i}}}^{2}, \\
\hat{y}_{I_{i}}=y_{I_{r_{i}}}+\sqrt{\frac{E_{i}}{E_{\mathrm{ST}\left|h_{i_{t}}\right|^{2}+\sigma_{n_{i_{t}}}^{2}}^{2}}} h_{r_{i}} y_{I_{i_{t}}} .
\end{gathered}
$$

Again in matrix form one has

$$
\mathbf{y}_{I}=\mathbf{h}_{I} s+\mathbf{n}_{I}+\mathbf{Y}_{I}
$$

where

$$
\begin{aligned}
& \mathbf{y}_{I}= {\left[\frac{1}{\sigma_{n_{r_{t}}}} y_{r_{t}} \frac{1}{\hat{\sigma}_{n_{r_{1}}}} y_{r_{1}} \cdots \frac{1}{\hat{\sigma}_{n_{r_{m}}}} y_{r_{m}}\right]^{T}, } \\
& \mathbf{h}_{I}=\left[\sqrt{\frac{E_{\mathrm{ST}}}{\sigma_{n_{r_{t}}}^{2}}} h_{r_{t}} \sqrt{\frac{E_{\mathrm{ST}} E_{1}}{\hat{\sigma}_{n_{r_{1}}}^{2}\left(E_{\mathrm{ST}}\left|h_{1_{t}}\right|^{2}+\sigma_{n_{n_{t}}}^{2}\right)}}\right. \\
& \times h_{r_{1}} h_{1_{t}} \cdots \sqrt{\frac{E_{\text {ST }} E_{m}}{\hat{\sigma}_{n_{r_{m}}}^{2}\left(E_{\mathrm{ST}}\left|h_{m_{t}}\right|^{2}+\sigma_{n_{m_{t}}}^{2}\right)}} \\
&\left.\times h_{r_{m}} h_{m_{t}}\right]^{T}, \\
& \mathbf{Y}_{I}= {\left[\frac{1}{\sigma_{n_{r_{t}}}} y_{I_{r_{t}}} \frac{1}{\hat{\sigma}_{n_{r_{1}}}} \hat{y}_{I_{1}} \cdots \frac{1}{\hat{\sigma}_{n_{r_{m}}}} \hat{y}_{I_{m}}\right]^{T}, }
\end{aligned}
$$

and $\mathbf{n}_{I} \sim \mathrm{CN}(\mathbf{0}, \mathbf{I})$.

2.2. Spectrum Sensing. Spectrum sensing is performed, by means of an energy detector, for the first $t_{s}$ seconds out of total time slot duration of $T$ seconds at the source node only. The remaining $T-t_{s}$ is used for transmission, after detecting a "spectral hole". The probabilities of detection and false alarm, according to [16], are given by

$$
\begin{gathered}
P_{d}=Q\left(\frac{\lambda-N-\gamma_{d}}{\sqrt{2\left(N+2 \gamma_{d}\right)}}\right), \\
P_{f}=Q\left(\frac{\lambda-N}{\sqrt{2 N}}\right),
\end{gathered}
$$

respectively, where $\lambda$ is the threshold of the energy detector, $N=t_{s} f_{s}$ is the number of samples, $f_{s}$ is the sampling frequency, $\gamma_{d}$ equals $N$ times the SNR at the output of the detector and $Q(\cdots)$ is the Gaussian $Q$-function.

\section{All Participate System}

In this section, an all-participate (AP) system is discussed. In such a system, all the relays forward the signal to the destination. Firstly, the optimization problem is formulated. Then the constraints on the objective function are derived. The SER at the destination is given by

$$
\begin{aligned}
\operatorname{SER}= & P\left(H_{0}\right)\left(Q\left(\sqrt{k \gamma_{0}}\right)\right)\left(1-P_{f}\right) \\
& +P\left(H_{1}\right)\left(Q\left(\sqrt{k \gamma_{I}}\right)\right)\left(1-P_{d}\right),
\end{aligned}
$$

where $\gamma_{0}$ is the SNR after combining, $\gamma_{I}$ is the signal-tointerference-plus-noise-ratio (SINR) after combining, $k$ is a constant which depends on the modulation scheme used, $P\left(H_{0}\right)$ is the probability of no primary user transmission, and $P\left(H_{1}\right)=1-P\left(H_{0}\right)$ is the probability of a primary user 
transmission. The SNR $\gamma_{0}$ can be found, assuming maximal ratio combining (MRC), as

$$
\gamma_{0}=\alpha+\sum_{i=1}^{m} \frac{\beta_{i}}{\tilde{\sigma}_{n_{r_{i}}}^{2}}
$$

where

$$
\alpha=\frac{p_{\mathrm{ST}} T_{s}\left|h_{r_{t}}\right|^{2}}{\sigma_{n_{r_{t}}}^{2}}, \quad \beta_{i}=\frac{p_{\mathrm{ST}} p_{i} T_{s}^{2}\left|h_{r_{i}}\right|^{2}\left|h_{i_{t}}\right|^{2}}{p_{\mathrm{ST}} T_{s}\left|h_{i_{t}}\right|^{2}+\sigma_{n_{i_{t}}}^{2}},
$$

where the source and relay energies have been replaced by

$$
E_{\mathrm{ST}}=p_{\mathrm{ST}} T_{s}, \quad E_{i}=p_{i} T_{S},
$$

where $p_{\mathrm{ST}}$ and $p_{i}$ s are the source and relay powers, respectively, and $T_{s}$ is the symbol time. Similarly, $\gamma_{I}$ can be expressed as

$$
\gamma_{I}=\frac{\left(\alpha+\sum_{i=1}^{m}\left(\beta_{i} / \hat{\sigma}_{n_{r_{i}}}^{2}\right)\right)^{2}}{\alpha+\sum_{i=1}^{m}\left(\beta_{i} / \hat{\sigma}_{n_{r_{i}}}^{2}\right)+\left(\alpha c / \sigma_{n_{r_{t}}}^{2}\right)+\sum_{i=1}^{m}\left(d_{i} / \hat{\sigma}_{n_{r_{i}}}^{2}\right)\left(\beta_{i} / \hat{\sigma}_{n_{r_{i}}}^{2}\right)},
$$

where

$$
\begin{gathered}
c=E\left[\left|y_{I_{r_{t}}}\right|^{2}\right], \\
d_{i}=E\left[\left|\hat{y}_{I_{i}}\right|^{2}\right], \\
E\left[\left|\hat{y}_{I_{i}}\right|^{2}\right]=E\left[\left|y_{I_{r_{i}}}\right|^{2}\right]+E\left[\left|y_{I_{i_{t}}}\right|^{2}\right]\left(\frac{E_{i}}{\left.E_{S T\left|h_{i_{t}}\right|^{2}+\sigma_{n_{i_{t}}}^{2}}\left|h_{r_{i}}\right|^{2}\right) .}\right.
\end{gathered}
$$

After substituting (15) and (18) in (14), the SER can be obtained as

$$
\begin{aligned}
\operatorname{SER} & =P\left(H_{0}\right) Q\left(\sqrt{k\left(\alpha+\sum_{i=1}^{m} \frac{\beta_{i}}{\tilde{\sigma}_{n_{r_{i}}}^{2}}\right)}\right) \\
& \times\left(1-Q\left(\frac{\lambda-N}{\sqrt{2 N}}\right)\right) \\
& +P\left(H_{1}\right)\left(1-Q\left(\frac{\lambda-N-\gamma_{d}}{\sqrt{2\left(N+2 \gamma_{d}\right)}}\right)\right) Q \\
& \times\left(\sqrt{\frac{k\left(\alpha+\sum_{i=1}^{m}\left(\beta_{i} / \hat{\sigma}_{n_{r_{i}}}^{2}\right)\right)^{2}}{\alpha+\sum_{i=1}^{m}\left(\beta_{i} / \hat{\sigma}_{n_{r_{i}}}^{2}\right)+\left(\alpha c / \sigma_{n_{r_{t}}}^{2}\right)+\sum_{i=1}^{m}\left(d_{i} / \hat{\sigma}_{n_{r_{i}}}^{2}\right)\left(\beta_{i} / \hat{\sigma}_{n_{r_{i}}}^{2}\right)}}\right) .
\end{aligned}
$$

Now we form the different constraints on the problem. First, we consider both individual power constraints at the source and the relay and a global power constraint on the whole system. Therefore, the constraints are given by

$$
\begin{gathered}
0 \leq p_{\mathrm{ST}} \leq p_{T}, \quad 0 \leq p_{i} \leq p_{i}^{\max }, \quad P_{f} \leq P_{f}^{\mathrm{th}}, \\
p_{\mathrm{ST}}+\sum_{i=1}^{m} p_{i} \leq p_{\text {total }}, \quad R T_{s}+t_{s} \leq T
\end{gathered}
$$

where $p_{\mathrm{ST}}$ is the power available at the source, $p_{i}^{\max }$ is the power available at each relay, $p_{\text {total }}$ is the power available to the whole system, and $P_{f}^{\text {th }}$ specifies the constraint on the probability of false alarm. The constraints on $P_{f}, T_{s}$, and $t_{s}$ are introduced to maintain an acceptable throughput. Next the two cases of global power constraint only and individual power constraints only are considered. For the case of global power constraint only, the constraints will be

$$
P_{f} \leq P_{f}^{\text {th }}, \quad p_{\mathrm{ST}}+\sum_{i=1}^{m} p_{i} \leq p_{\text {total }}, \quad R T_{s}+t_{s} \leq T .
$$

In the other scenario, the constraints are given by

$$
\begin{gathered}
0 \leq p_{\mathrm{ST}} \leq p_{T}, \quad 0 \leq p_{i} \leq p_{i}^{\max }, \quad P_{f} \leq P_{f}^{\text {th }}, \\
R T_{s}+t_{s} \leq T .
\end{gathered}
$$

The individual power constraints are set to limit the interference suffered by the primary user in the case of missed detection. As there is no individual power constraint, the interference caused to the user in the global power constraint only case, where the primary user is only protected by spectrum sensing, is greater.

The problem with optimizing (22) is that it is a nonlinear and nonconvex function due to the Gaussian Q-function being nonlinear and, in general, nonconvex. Thus, the Lagrangian multiplier method [17] cannot be applied here to obtain closed form expressions of the optimal resource allocation. One has to resort to numerical techniques to obtain the optimal solution.

A special case of importance is the absence of the direct link between source and destination, because the relays take on a more prominent role in the presence of no direct link. In this case, the SER is can be obtained by setting $\alpha=0$ in (22).

\section{Selective Relaying}

The drawback of the all-participate (AP) scheme discussed in the previous section is that to avoid causing interference, the source and the relay transmit on orthogonal channels. Hence, consuming a considerable amount of resources. In our discussion of a time orthogonal systems, $m+1$ time slots are utilized for the transmission of one data frame. Additionally, as no sensing is performed at the relays, the primary may become active over any one of the $m$ time slots and cause interference.

To overcome these problems, a selection scheme is proposed in this section in which only one relay is selected to take part in forwarding the signal from the source. Now only 2 time slots are used in transmitting one frame of data and thus decreasing the likelihood of primary becoming active again during relay transmission. In the selection case, the SER is

$$
\begin{aligned}
\operatorname{SER}= & P\left(H_{0}\right) Q\left(\sqrt{k\left(\alpha+\frac{\beta_{j}}{\widetilde{\sigma}_{n_{r_{j}}}^{2}}\right)}\right)\left(1-Q\left(\frac{\lambda-N}{\sqrt{2 N}}\right)\right) \\
& +P\left(H_{1}\right) Q
\end{aligned}
$$




$$
\begin{aligned}
& \times\left(\sqrt{\frac{k\left(\alpha+\left(\beta_{j} / \widehat{\sigma}_{n_{r_{j}}}^{2}\right)\right)^{2}}{\alpha+\left(\beta_{j} / \widehat{\sigma}_{n_{r_{j}}}^{2}\right)+\left(\alpha c / \sigma_{n_{r_{j}}}^{2}\right)+\left(d_{j} / \widehat{\sigma}_{n_{r_{j}}}^{2}\right)\left(\beta_{j} / \widehat{\sigma}_{n_{r_{j}}}^{2}\right)}}\right) \\
& \times\left(1-Q\left(\frac{\lambda-N-\gamma}{\sqrt{2(N+2 \gamma)}}\right)\right),
\end{aligned}
$$

where $b_{j}, d_{j}$, and $\sigma_{n_{r_{j}}}^{2}$ correspond to the $j$ th relay that is selected. The SER in (26) is first optimized for all the relays and the relay which gives the minimum optimum SER is selected. Again, all three cases given in (23), (24) and (25) of both global and individual constraints, global constraint only and individual constraint only are considered. The selection criteria of minimizing SER adds complexity. However, such a criteria provides results which can serve as a benchmark as minimizing SER is the optimal selection criteria.

It is again evident that, even in the selection case, the SER is still a nonlinear and nonconvex function. Therefore, one has to resort to numerical techniques to find the optimal solution. The special case of no direct link is again of particular interest and considered separately.

\section{Numerical Results}

In this section, numerical results are provided for the optimization problems discussed. First, the proposed AP system with optimal resource allocation is discussed and it is shown that the proposed AP schemes give better performance than the uniform power allocation (UPA) scheme. In UPA, the power is uniformly distributed among the source and the relays and the sensing time and the symbol time are set so that the inequality $R T_{s}+t_{s} \leq T$ is satisfied. The selection scheme is discussed next and its performance is compared with selection with UPA. To make it easy for the reader to follow the discussion, a glossary is included in Table 1 .

An interior-point algorithm was used to perform the optimization. The MATLAB function fmincon, which performs constrained optimization, is used to run the interiorpoint algorithm. To ensure that the algorithm converged to the optimal solution, the algorithm was run for a large number of initial values. All the variances are set as equal, $\sigma_{n_{r_{t}}}^{2}=\sigma_{n_{i_{t}}}^{2}=\sigma_{n_{r_{i}}}^{2}=\sigma^{2}$. The constraint of $P_{f}$ is set at $P_{f}^{\text {th }}=0.1$. The total time duration is taken to be $100 \mathrm{~ms}$. Binary phase shift keying (BPSK) is the modulation scheme employed. Due to the the number of samples and the sensing time being linearly related, the results are plotted against the number of samples.

The relationship between the number of samples $(N)$ and SER is shown in Figure 2. As one can clearly observe from Figure 2, there is an optimal value of the number of samples, hence for the sensing time, which minimizes the SER. This is because of the tradeoff between symbol time, $T_{s}$ and sensing time, $t_{s}$. Increasing sensing time means higher probability of detection which leads to a lower SER. However, an increase in sensing time comes at the cost of a decrease in symbol time which leads to lower $\gamma_{0}$ and $\gamma_{I}$. Therefore, the SER

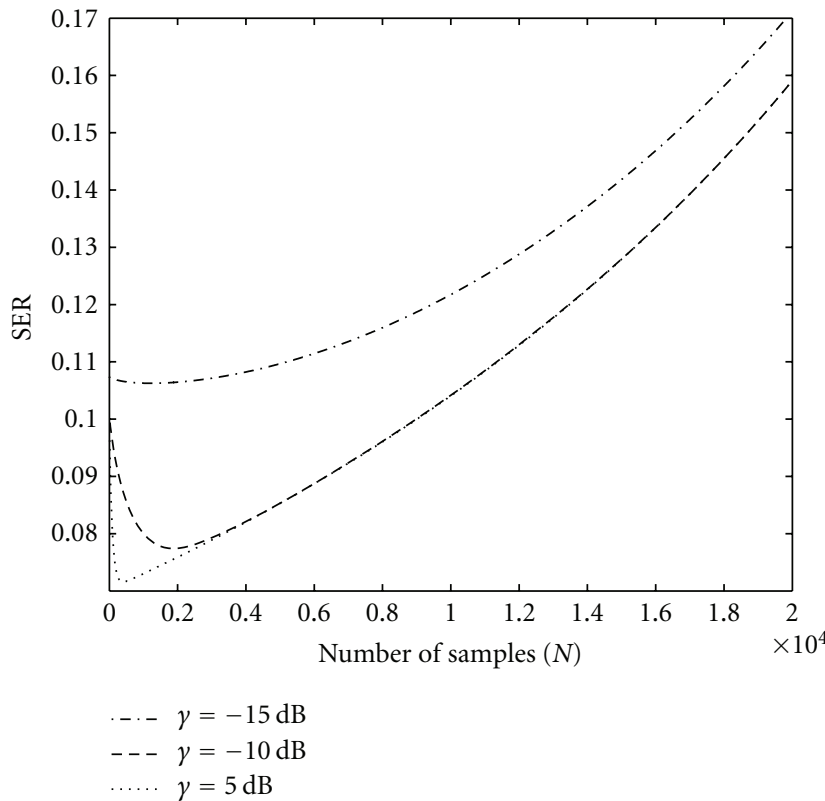

Figure 2: SER as a function of the number of samples $(N)$.

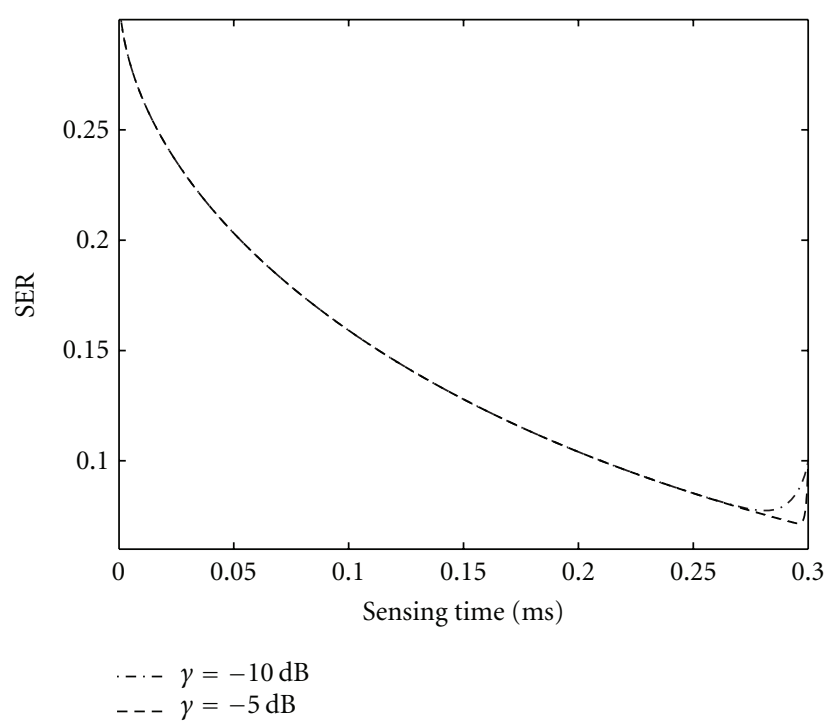

FIGURE 3: SER as a function of the symbol time $\left(T_{s}\right)$.

increases. Similarly, decreasing sensing time implies a lower probability of detection and, in turn, higher SER. However, it also means high values of $\gamma_{0}$ and $\gamma_{I}$ due to increase in symbol time, which in turn leads to lower SER. Hence, there exists an optimal value. This optimal value is affected by the primary user's SNR. The higher the primary user's SNR, the lower the optimal value of the sensing time will be as it takes shorter time to reach the same value of $P_{d}$ as it would take for a lower SNR of the primary user.

Figure 3 shows the relationship between the symbol time, $T_{s}$, and the SER. The relationship follows the opposite pattern as the sensing time. This is due to the constraint relating sensing time and the symbol time. Therefore, when 
TABLe 1: Glossary.

\begin{tabular}{ll}
\hline Acronym & Full name \\
\hline AP-ORA & All-participate with optimal resource allocation \\
AP-ORA-GL & All-participate optimal resource allocation with global constraint only \\
AP-ORA-Ind & All-participate optimal resource allocation with individual constraints only \\
UPA & uniform power allocation \\
AP-ORA-NDL & All-participate optimal resource allocation with no direct link \\
AP-ORA-GL-NDL & All-participate optimal resource allocation with global constraint only and no direct link \\
AP-ORA-Ind-NDL & All-participate optimal resource allocation with individual constraints only and no direct link \\
UPA-NDL & uniform power allocation with no direct link \\
Sel-ORA & selection with optimal resource allocation \\
Sel-ORA-GL & selection optimal resource allocation with global constraint only \\
Sel-ORA-Ind & selection optimal resource allocation with individual constraints only \\
Sel-UPA & selection with uniform power allocation \\
Sel-ORA-NDL & selection optimal resource allocation with no direct link \\
Sel-ORA-GL-NDL & selection optimal resource allocation with global constraint only and no direct link \\
Sel-ORA-Ind-NDL & selection optimal resource allocation with individual constraints only and no direct link \\
Sel-UPA-NDL & selection uniform power allocation with no direct link \\
\hline
\end{tabular}

the optimal value of the sensing time is low, the optimal value of the symbol time is high.

Figure 4 shows the SER performance of the different AP schemes plotted against $\gamma_{s}$, where $\gamma_{s}=1 / \sigma^{2}$. As expected, for the case with a direct link, the three optimal resource allocation (ORA) scenarios, global constraint only (GL), individual constraints only (Ind), and both global and individual constraints, outperform the uniform power allocation (UPA) and the direct link only for all values of $\gamma_{s}$ and the gap in performance becomes wider with increasing $\gamma_{s}$.

In Figure 4, in the case where there is no direct link (NDL) between source and destination, the performance is a little different. In this case, all three ORA schemes, AP-ORA-NDL, AP-ORA-GL-NDL, and AP-ORA-Ind-NDL, outperform the uniform power allocation scheme (UPANDL). However, for $\gamma_{s}$ less than $0 \mathrm{~dB}$, the direct link only case provides better SER performance than the three ORA cases with no direct link. The three ORA schemes with no direct link start to catch up to the direct link only scenario after $0 \mathrm{~dB}$ and completely outperform it after $5 \mathrm{~dB}$. This phenomena coupled with the fact that AP-ORA-NDL, AP-ORA-GLNDL, and AP-ORA-Ind-NDL, are handily outperformed by AP-ORA, AP-ORA-GL, and AP-ORA-Ind, respectively, demonstrate the significance of the presence of a link between the source and destination.

Comparing the different constraints, AP-ORA gives the worst performance in both scenarios of direct and no direct link. This is due to the fact that AP-ORA is constrained both globally and individually. Thus, even if one relay has more favourable conditions, the power allocated to it cannot exceed $p_{i}^{\max }$ which is not the case in global constraint only case. In global constraint only case more power can be allocated to the source and relay which has more favourable conditions. The comparison between global constraint only and individual constraints only requires further elaboration.

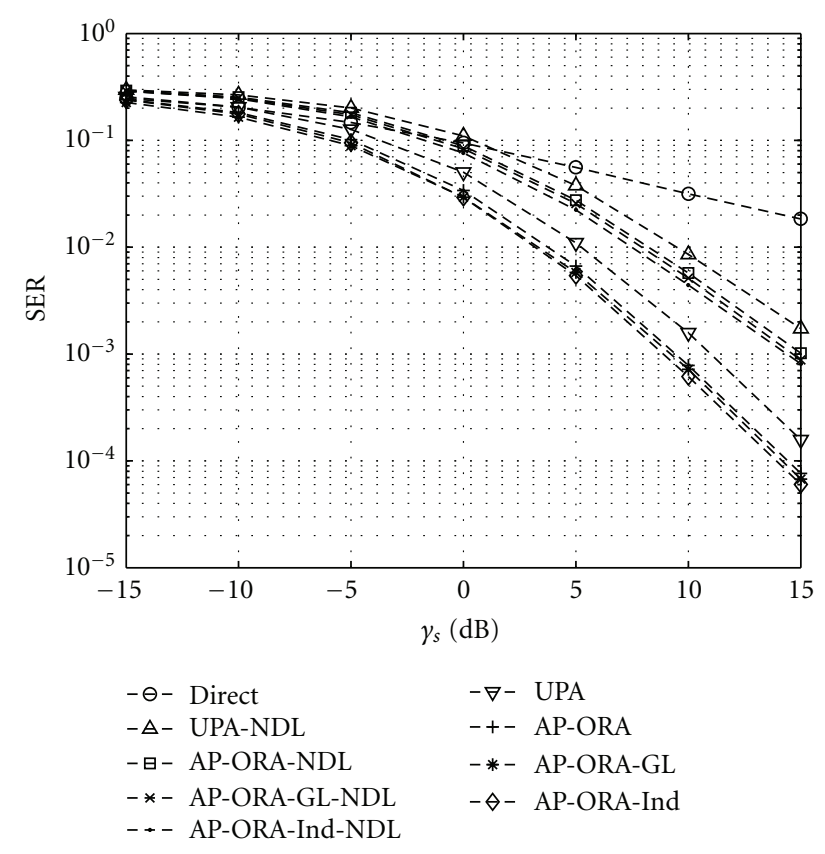

FIGURE 4: Comparison of SER performance of an all-participate under different schemes and constraints with $m=3$.

First, the global constraints only and individual only scenarios are compared in the no direct link case. Here, AP-ORA-Ind-NDL provides lower SER than AP-ORA-GLInd for all values of $\gamma_{s}$. AP-ORA-GL-NDL has the advantage of allocating more power to relays with better channel conditions. However, AP-ORA-Ind-NDL makes up for this advantage by having more total power in the system as it is not constrained by a total power constraint. 


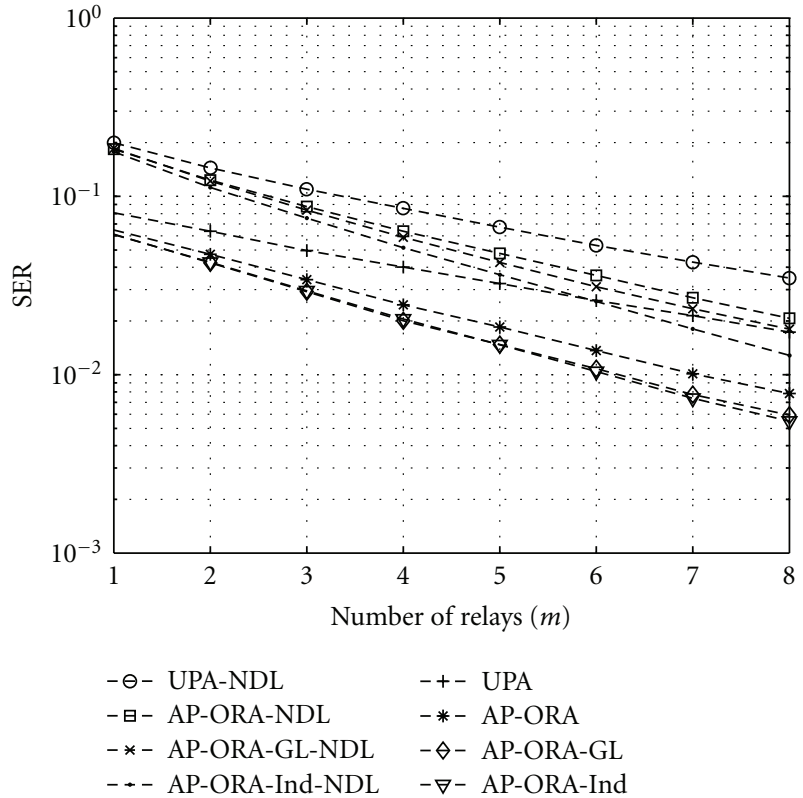

FIGURE 5: SER as a function of number of layers $(m)$ for the AP system.

Now consider the direct link case. Here, AP-ORA-GL outperforms AP-ORA-Ind at low values of $\gamma_{s}$ due to the presence of the direct link. As discussed before, the direct link is quite important, hence, as AP-ORA-GL is not limited by individual constraints, more power can be allocated to the direct link. This is not the case for AP-ORA-Ind. Therefore, AP-ORA-GL gives lower SER. However, with an increase in $\gamma_{s}$, the noise decreases and the greater total power in the case of AP-ORA-Ind comes more into play. Thus, APORA-Ind starts to outperform AP-ORA-GL at higher values of $\gamma_{s}$. However, we must keep in mind that in the global power constraint only case, the interference to the primary is greater than those in the other two cases. Hence, the advantage in performance at low $\gamma_{s}$ comes at the cost of greater interference to the primary.

Figure 5 shows the SER performance of the AP system as a function of the number of relays, $m$. A similar pattern to Figure 4 is observed. The ORA schemes outperform the UPA schemes in both cases of direct and no direct link. Among the proposed ORA schemes, AP-ORA-Ind-NDL provides lower SER than AP-ORA-NDL and AP-ORA-GL-NDL in the no direct link scenario while in the direct link scenario APORA is outperformed by both AP-ORA-Ind and AP-ORAGL. In addition, AP-ORA-GL has better performance than AP-ORA-Ind for a small number of relays. However, as the number of relays increases AP-ORA-Ind surpasses AP-ORAGL in terms of performance due to greater total power. Moreover, AP-ORA-NDL, AP-ORA-GL-NDL, and AP-ORAInd-NDL even start to outperform UPA for a large number of relays which shows the gain in performance due to ORA.

Figure 6 shows the performance of the various selection schemes as a function of $\gamma_{s}$. The comparison in performance follows a similar pattern as in the AP case with the proposed

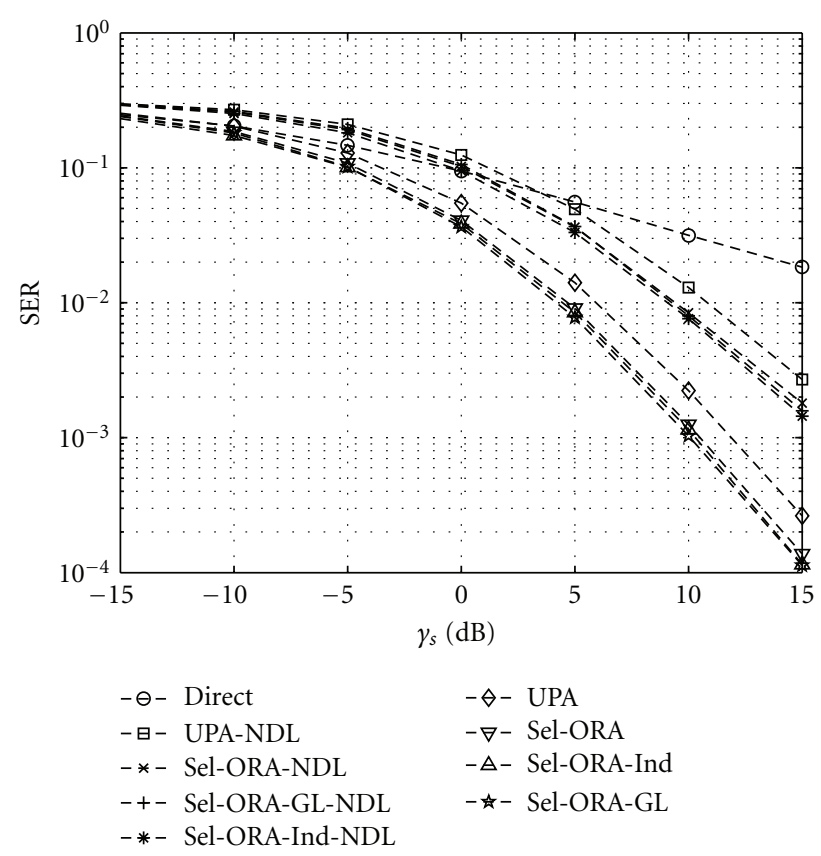

FIGURE 6: Comparison of SER performance of a selection system under different schemes and constraints with $m=3$.

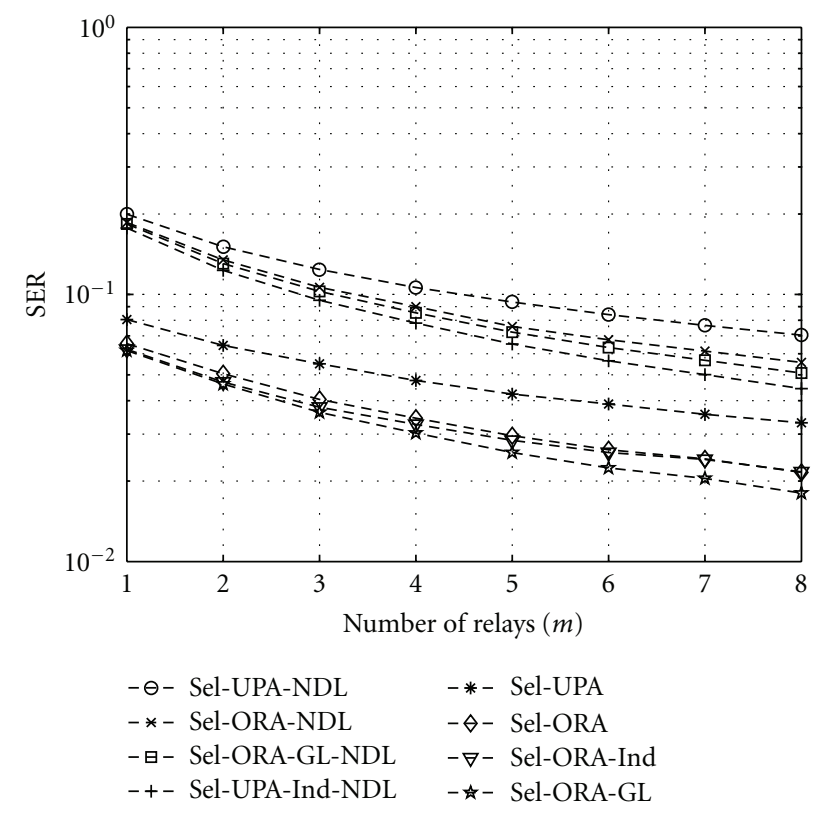

FIGURE 7: SER as a function of number of relays $(m)$ for selective relaying.

selection ORA schemes outperforming their UPA counterparts and direct link only scenario. However, there is one major difference. In the presence of a direct link, Sel-ORAInd gives poorer performance than Sel-ORA-GL even for high values of $\gamma_{s}$. Only at around $15 \mathrm{~dB}$ does Sel-ORAInd starts to catch up to Sel-ORA-GL. This is due to the fact that as pointed out in the AP system, in the case of global constraints only more power can be allocated to the 


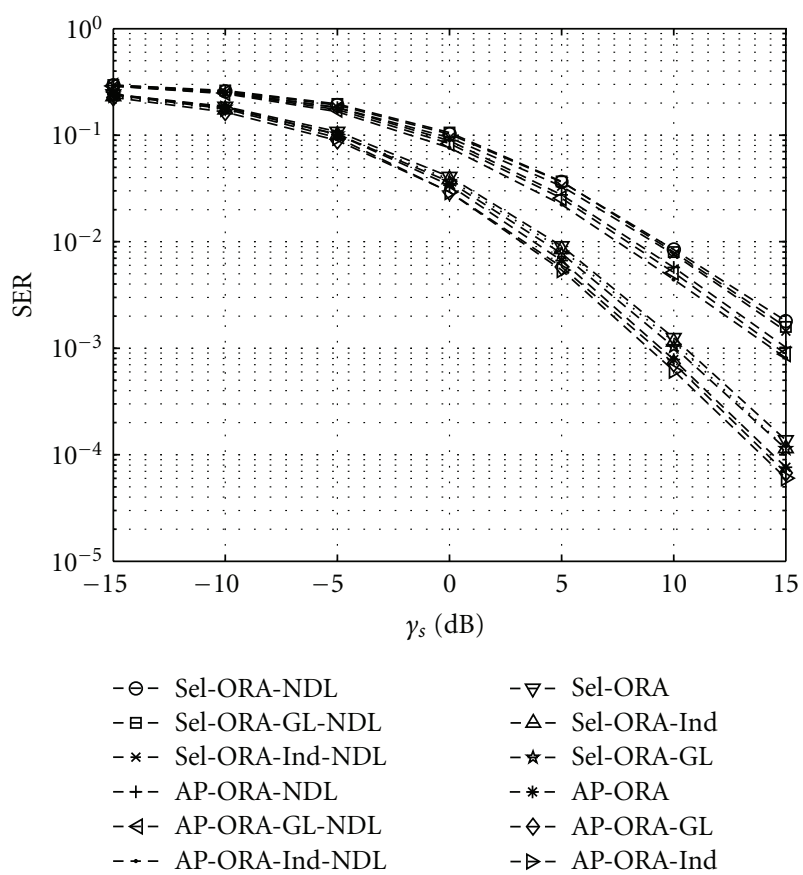

Figure 8: Comparison of AP vs Sel with $m=3$.

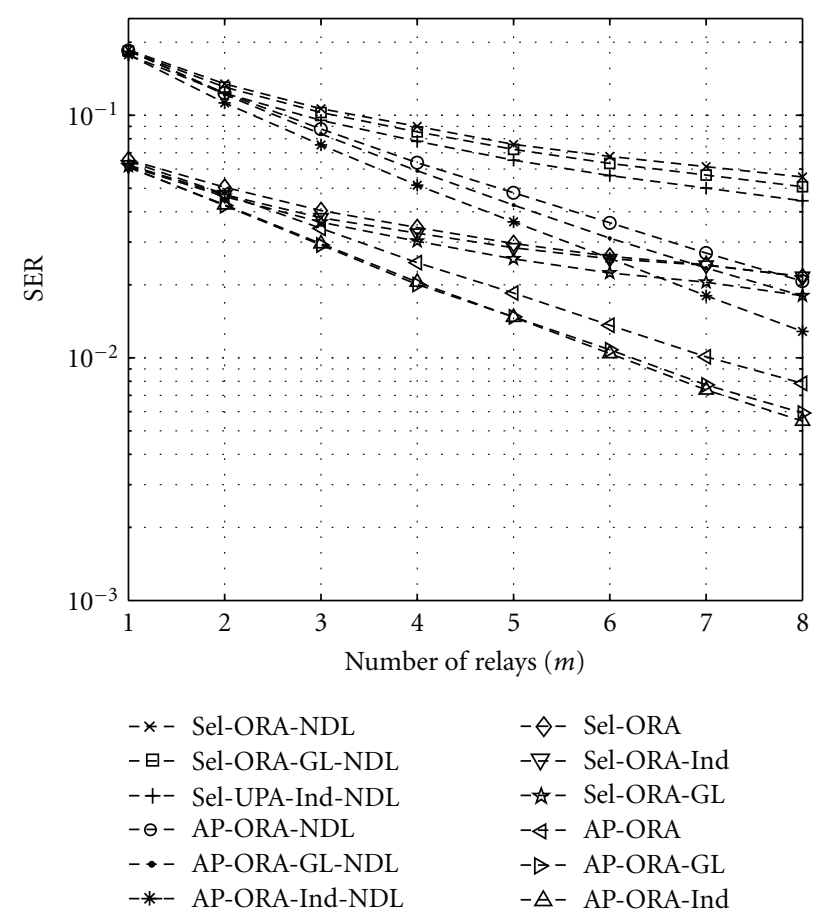

FIgURE 9: Comparison of AP versus Sel as a function of $m$.

\section{Conclusions}

source. However, unlike AP, in Sel there is only one additional relay which implies less total power for Sel-ORA-Ind and, therefore, requires a high value of $\gamma_{s}$ to make the difference in total power count.

SER performance for selective relaying as a function of the number of relays is shown in Figure 7. Again, the main difference from the AP case is that Sel-ORA-GL outperforms Sel-ORA-Ind even for a large number of relays. This is due to the fact that even though the number of relays increase, the total power for Sel-ORA-Ind remains constant as only one relay in addition to the source takes part in data transmission. An interesting point to note here is that the there seems to be a minimum threshold for the SER for the selective system.

Figures 8 and 9 show the performance comparison between the AP and Sel system as a function of $\gamma_{s}$ and $m$, respectively. The comparison is presented separately for clarity, as if it was included in the previous figures, they would have become cluttered. From Figure 8, one can see that AP scheme outperforms the selection scheme in all scenarios, however, the gap in performance is not too big. This is due to the fact that the total number of relays is 3 . If $m$ is increased, the performance gap will also increase. Still, one has to keep in mind the extra cost and spectral inefficiency associated with the AP scheme. This becomes more clear when the Figure 9 is examined.

As one can see, the difference in performance between the respective AP schemes and Sel schemes increases with increase in number of relays. As discussed earlier, the Sel schemes look to be bounded by a minimum threshold. Due to this, Sel with direct link scenarios even fall below the AP with no direct link scenarios for a large number of relays.
In this paper, ORA for a cognitive relay network has been discussed. It has been shown that for an AP system that ORA improves SER performance and the discussed schemes outperform the UPA schemes. The importance of the direct link between the source and the destination has also been demonstrated. Among the different constraints on the system, the case of both individual and global constraints gives the worst performance while global constraints only is the best for low $\gamma_{s}$. However, this comes at the cost of greater interference to the primary user. The individual constraints only case takes over as the best scheme as $\gamma_{s}$ increases.

It was then noted that the AP scheme consumes considerable resources and is spectrally inefficient. Therefore, a simple relay selection scheme has been proposed. Optimal resource allocation was then discussed for the selection scheme. The performance comparison of the AP and Sel shows that while AP provides better SER performance, it comes at the cost of considerable resources.

\section{Acknowledgment}

This work was supported by King Abdullah University of Science and technology (KAUST).

\section{References}

[1] "Federal communications comission (fcc), et docket no 03322 notice of proposed rule making and order," 2003.

[2] J. Mitola and G. Maguire Jr., "Cognitive radio: making software radios more personal," IEEE Personal Communications, vol. 6, no. 4, pp. 13-18, 1999. 
[3] S. Haykin, "Cognitive radio: brain-empowered wireless communications," IEEE Journal on Selected Areas in Communications, vol. 23, no. 2, pp. 201-220, 2005.

[4] Y. Liang, Y. Zeng, E. C. Y. Peh, and A. T. Hoang, "Sensingthroughput tradeoff for cognitive radio networks," IEEE Transactions on Wireless Communications, vol. 7, no. 4, pp. 1326-1337, 2008.

[5] S. Stotas and A. Nallanathan, "Optimal sensing time and power allocation in multiband cognitive radio networks," IEEE Transactions on Communications, vol. 59, no. 1, pp. 226$235,2011$.

[6] J. Laneman, D. Tse, and G. Wornell, "Cooperative diversity in wireless networks: efficient protocols and outage behavior," IEEE Transactions on Information Theory, vol. 50, no. 12, pp. 3062-3080, 2004.

[7] W. Yue, B. Zheng, and Q. Meng, "Optimal power allocation for cognitive relay networks," in Proceedings of the International Conference on Wireless Communications and Signal Processing (WCSP '09), pp. 1-5, Nanjing, China, November 2009.

[8] L. Li, X. Zhou, H. Xu, G. Y. Li, D. Wang, and A. Soong, "Simplified relay selection and power allocation in cooperative cognitive radio systems," IEEE Transactions on Wireless Communications, vol. 10, no. 1, pp. 33-36, 2011.

[9] J. Mietzner, L. Lampe, and R. Schober, "Distributed transmit power allocation for multihop cognitive-radio systems," IEEE Transactions on Wireless Communications, vol. 8, no. 10, pp. 5187-5201, 2009.

[10] Z. Liu, Y. Xu, D. Zhang, and S. Guan, "An efficient power allocation algorithm for relay assisted cognitive radio network," in Proceedings of the International Conference on Wireless Communications and Signal Processing (WCSP '10), pp. 1-5, Suzhou, China, October 2010.

[11] X. Liu, B. Zheng, J. Cui, and W. Ji, "A new scheme for power allocation in cognitive radio networks based on cooperative relay," in Proceedings of the 12th IEEE International Conference on Communication Technology (ICCT '10), pp. 861-864, Tsukuba Science City, Novmber 2010.

[12] X. Qiao, Z. Tan, S. Xu, and J. Li, "Combined power allocation in cognitive radio-based relay-assisted networks," in Proceedings of the IEEE International Conference on Communications Workshops (ICC '10), pp. 1-5, Cape Town, South Africa, May 2010.

[13] X. Liu, B. Zheng, and W. Ji, "Cooperative relay with power control in cognitive radio networks," in Proceedings of the 6th International Conference on Wireless Communications, Networking and Mobile Computing (WiCOM '10), pp. 1-5, Chengdu, China, September 2010.

[14] L. Jayasinghe and N. Rajatheva, "Optimal power allocation for relay assisted cognitive radio networks," in Proceedings of the IEEE 72nd Vehicular Technology Conference Fall (VTC2010-Fall '10), pp. 1-5, Ottawa, Canada, September 2010.

[15] Z. Shu and W. Chen, "Optimal power allocation in cognitive relay networks under different power constraints," in Proceedings of the IEEE International Conference on Wireless Communications, Networking and Information Security (WCNIS '10), pp. 647-652, Beijing, China, June 2010.

[16] H. Urkowitz, "Energy detection of unknown deterministic signals," Proceedings of the IEEE, vol. 55, no. 4, pp. 523-531, 1967.

[17] S. Boyd and L. Vandenberghe, Convex Optimization, Cambridge University Press, 2004. 

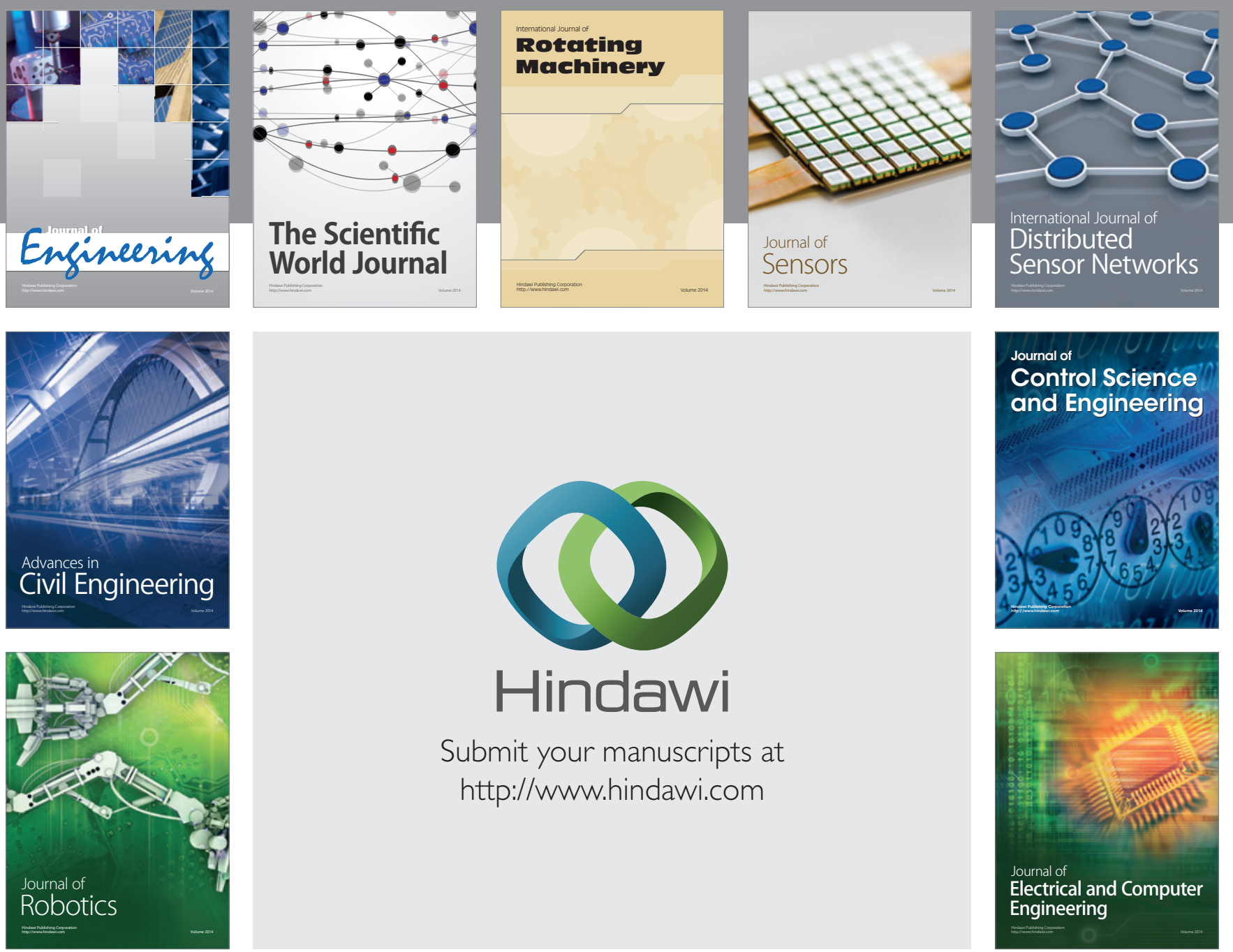

Submit your manuscripts at

http://www.hindawi.com
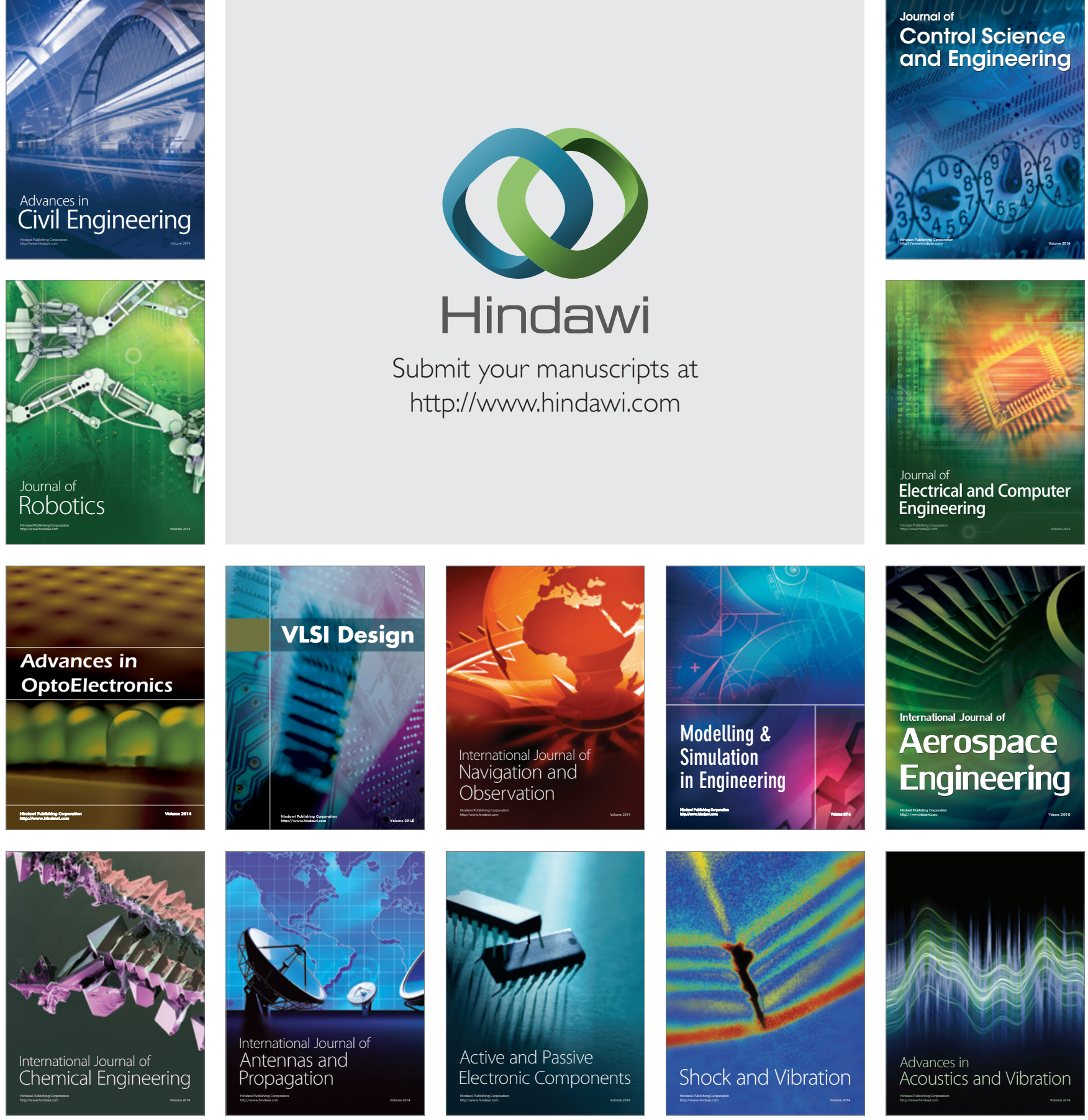